\title{
A Barometric Survey of Dust-Devil Vortices on a Desert Playa
}

\section{Ralph D. Lorenz · Peter D. Lanagan}

\begin{abstract}
Dust devils, and other columnar vortices, are associated with local surface pressure drops that can be observed in time-series data on both Earth and Mars. High cadence measurements are needed to resolve these small structures, and we report a month-long survey (June/July 2012) on a Nevada desert playa using microbarographs sampled multiple times per second. Candidate dust-devil signatures are classified, with detections being robust at about one per day for pressure drops exceeding $0.3 \mathrm{hPa}$ (roughly a 5:1 signal-to-noise threshold, where the observed noise level corresponds reasonably well with the dynamic pressure associated with the estimate convective velocity scale). The vortex population is evaluated and compared with those observed on Mars : a broken power law or a more convex distribution describes the terrestrial data. A single station observes about three events per week (for normalized pressure drops of $0.06 \%$ ), about three times fewer than Mars observations for the same normalized drop. We find evidence for clustering of vortex events in a pseudoperiodic manner with a 20-min period, consistent with the size of boundary-layer convection cells.
\end{abstract}

Keywords : Cellular convection · Dust devils · Pressure sensing · Vortices

RD Lorenz, Johns Hopkins University Applied Physics Laboratory, 11100 Johns Hopkins Road, Laurel, MD 20723, USA.

*Corresponding Author. Tel. +1 4437782903 Fax. +1 4437788939 Email: Ralph.lorenz@jhuapl.edu

PD Lanagan, Department of Physical Science, College of Southern Nevada, 700 College Dr., Henderson, NV 89002 


\section{Introduction}

While pressure drops associated with dust devils have been recorded on Earth (e.g., Wyett, 1954; Lambeth, 1966; Sinclair, 1973), they are actually more systematically documented in studies of dust devils on Mars (e.g., by Mars Pathfinder: Murphy and Nelli, 2002, and by the Phoenix mission, Ellehoj et al., 2010), where landers have recorded meteorological parameters over long periods with a high enough cadence to detect small vortical structures. Most terrestrial meteorological records have cadence too low (canonically, $15 \mathrm{~min}$ ) to record dust devils, for which a sampling rate of $\approx 1 \mathrm{~Hz}$ or better is typically required. On the other hand, field surveys to observe dust devils with in-situ meteorological data have typically been too short to acquire a meaningful number of events (the survey by Lambeth 1966, a halfcentury ago, is the largest, with only 19 events), or have been made with mobile 'chase' platforms that do not yield the same unbiased survey that a fixed platform may (see also Lorenz, 2012a).

The peak pressure drops measured by the Phoenix and Pathfinder landers on Mars (recording $\approx 500$ and $\approx 80$ events respectively, over about 100 days in each case) appear to follow a power-law population function, specifically, a -2 power differential distribution, Lorenz (2012a). The previous terrestrial data do not permit a statistical discrimination of candidate population functions, but do not exclude the same relationship.

We have reported previously on the utility of new pressure logging instrumentation (Lorenz, 2012b) for undertaking this kind of measurement, and also noted that while a simple Gaussian or Lorentzian (Ellehoj et al., 2010) 'lineshape' signature occurs for constant velocity encounters, a diverse range of skewed or multi-pole signatures can result from the cycloidal path of a devil nearby (Lorenz, 2013), even for singlecore vortices. Here we examine a continuous high-resolution pressure record to provide a census of vortices on Earth at a location known for dust-devil activity (e.g. Pathare et al., 2010; Metzger et al., 2011; Balme et al., 2013), although it should be considered that such an approach detects pressure drops from all vortices without reference to whether they are dust-laden or not. Because the survey is made post hoc on the pressure time series, it is directly analogous to the surveys conducted on Mars (Murphy and Nelli, 2002; Ellehoj et al., 2010). In addition to being a convenient measure of the overall intensity of a dust devil (as is also the case with other vortex motions such as tornados, e.g. Karstens et al., 2010), the pressure drop may itself play a significant role in dust-lifting (e.g. Balme and Hagermann, 2006).

\section{Instrumentation and Location}

Our measurements were made with field pressure loggers (Lorenz, 2012b) each with a plastic case that houses 2 D-cell alkaline batteries and a Gulf Coast Data Concepts B1100 pressure logger, which stores data from a precision Bosch BMP085 pressure sensor (logged with a resolution of $1 \mathrm{~Pa}$ ) as ASCII files on a flash memory card. The unit can operate for months at sample rates of $2 \mathrm{~Hz}$ or more. The field site is a desert playa in southern Nevada that has been the subject of previous surveys of dust-devil activity (e.g. Pathare et al., 2010; Balme et al., 2012; Metzger et al., 2012). The playa is adjacent to route 95 in a basin to the south-east of Las Vegas (see Fig.1).

Three measurement stations were deployed between June 11 and July 11 2012. Two (P11 and P10B) were deployed towards the eastern side of the main part of the playa (see Fig. 1), whereas a third was deployed on the alluvial fan studded with creosote bush that marks the western margin of the playa proper. 
Context meteorological data are available from the Community Environmental Monitoring Program meteorological station CMP04 at Boulder City, $35.985{ }^{\circ} \mathrm{N} 114.84139{ }^{\circ} \mathrm{W}$. This station, at an elevation of $735 \mathrm{~m}$, is $16 \mathrm{~km}$ from our survey location (see Fig.1), but across open scrubby desert and thus can be considered broadly representative of conditions at the playa. There is also a weather station, although with longer data gaps, at the Boulder City municipal airport, about $2 \mathrm{~km}$ closer. In both cases, with typical winds from the south of $5 \mathrm{~m} \mathrm{~s}^{-1}$, an air parcel will be advected near the weather station about 30 min after it passes the playa. The weather data show conditions that are typical of early desert summer, with high daytime temperatures (approximately $40{ }^{\circ} \mathrm{C}$ ), low humidity with wind from the south and strong solar heating. Average windspeeds are $3-5 \mathrm{~m} \mathrm{~s}^{-1}$ in the early afternoon, which is the time for peak dustdevil activity. This wind-speed range is favourable for dust-devil formation - speeds in excess of $7 \mathrm{~ms}^{-1}$ tend to suppress activity.

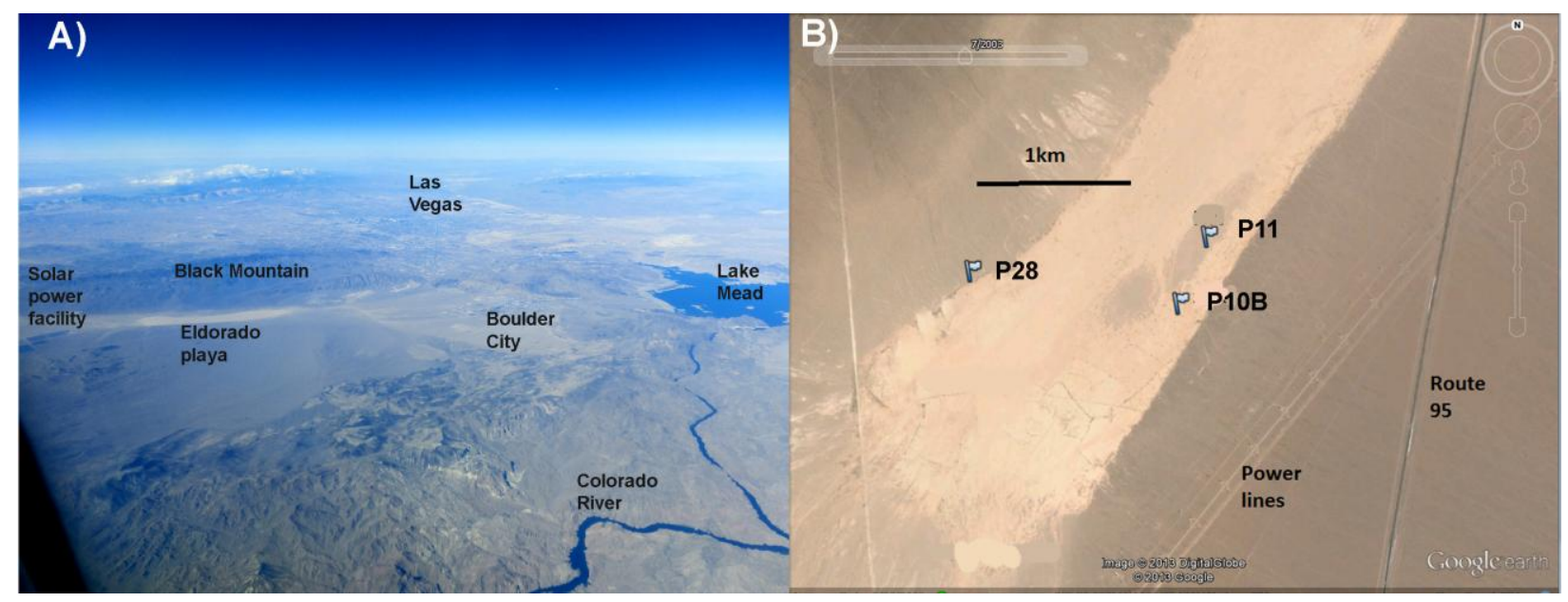

Fig 1. (A) Oblique view from a commercial airliner looking west-north-west, showing the study site, the prominent bright playa in the Eldorado basin between Black Mountain and the Eldorado mountains in the foreground. Boulder City, Nevada is $\approx 15 \mathrm{~km}$ away from the playa and is at the basin's northern end, where hills separate it from Lake Mead and Las Vegas. (B) Vertical view of the playa from Google Earth, showing the locations of the three measurement stations. 


\section{Observations and Event Identification}

High resolution microbarograph records show a variety of atmospheric phenomena. In addition to the diurnal cycle and the familiar rising or falling associated with synoptic weather systems, gravity waves can be detected (e.g. Johnson, 1929), as well as the signatures of distant volcanic eruptions (famously, Krakatoa), nuclear explosions (e.g. Posey and Pierce, 1971) or bolide entries (e.g. that over Chelyabinsk in 2013). Under some conditions, pressure fluctuations with periods of 200 to $1000 \mathrm{~s}$ are associated with pseudoperiodic structures in the atmospheric boundary layer associated with convection cells (Frisch et al., 1976). Such pressure fluctuations have been found also in large eddy simulations of the boundary layer (e.g. Spiga, 2012) and dust-devil vortices tend to be associated with the upwelling legs of the convection cell pattern (e.g. Kanak, 2005; Toigo et al., 2003).

In our data, a typical day's record shows three principal phenomena (Fig. 2): first is the diurnal variation, with a peak-peak amplitude of about $7 \mathrm{hPa}$. Some parts of the day show scatter of only about $0.2 \mathrm{hPa}$, whereas some parts (notably around dawn) have markedly broader symmetric variations of over $0.5 \mathrm{hPa}$ (Fig. 2b). It is suspected these are associated with turbulence generated as the nocturnal low-level jet is mixed back down to the surface. In the afternoon, very localized pressure dips can be noticed, associated with convective vortices (which may or may not be dust-laden).
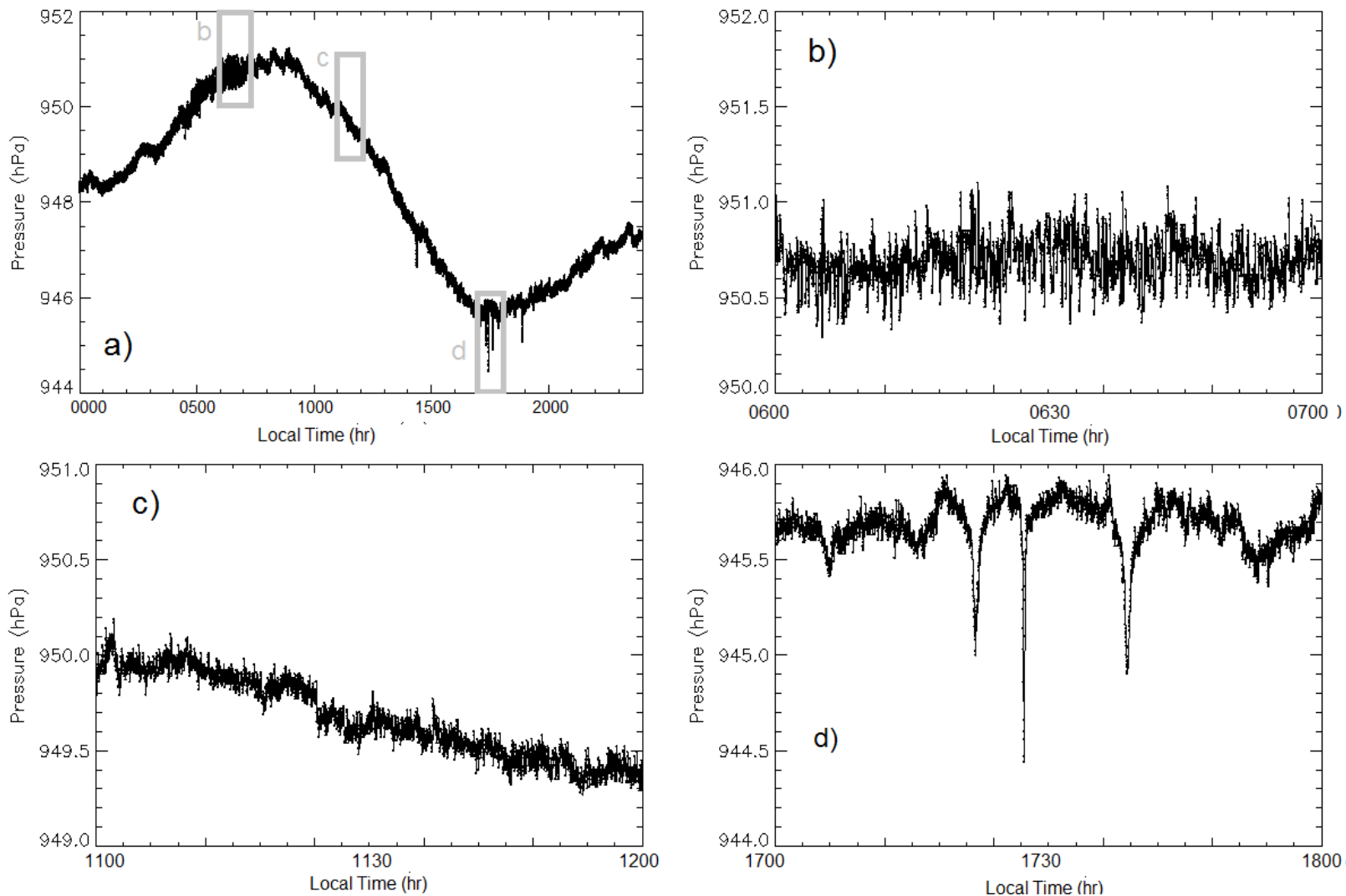
Submitted to Boundary Layer Meteorology, 13 January, 2014. Accepted 15 July 2014

Fig 2. A 24-hr pressure record (a) with zoom of three highlighted areas. On some days a distinctive highfrequency fluctuation period develops around dawn (b) with a $0.6 \mathrm{hPa}$ peak-to-peak amplitude: see text for a possible explanation.Quiescent period (c) in the mid-morning shows noise or pressure fluctuations of about $0.1 \mathrm{hPa}$, which may reflect the noise level of the instrument. In the afternoon the distinctive dustdevil pressure drops are seen (d). Note the pseudoperiodic recurrence of dips in the pressure trace.

The month-long dataset was examined by plotting data at an expanded scale in roughly 1-hr segments : a script in the Interactive Data Language (IDL) was written to allow the dataset to be efficiently stepped through, with a mouse click to advance to the next hour of data, or to designate a feature for future study. These candidate dust-devil features (about 200 in each dataset) were subsequently viewed more closely by rescaling the plot over a $2 \mathrm{hPa}$ range and over a 5 minute window and classified as, $\mathrm{A}$ - certain dust devil, B - probable dust devil, or C - possible dust devil. These subjective classifications were as follows: for A, a clear history of sharp pressure decline and recovery, well in excess of nearby pressure excursions, was noted. For B, a pressure drop was apparent, but may have been only modestly larger than background variations and/or had a discontinuity or other features not characteristic of a classical vortex passage. For $\mathrm{C}$ events (not shown), closer inspection rejected the classification, typically because other nearby fluctuations may have been comparable with the suspected event.

Examples of the A and B classifications are given in Figs. 3 and 4. Any classification scheme is necessarily subjective, and there are of course borderline cases where a signature might be classed as A or $\mathrm{B}$, or as B or C. There is, however, no uncertainty in class $\mathrm{C}$ events being misdesignated as class A or vice versa. Thus any automated classification scheme should aim to detect all Class A events, and some or most of Class B, but no others.
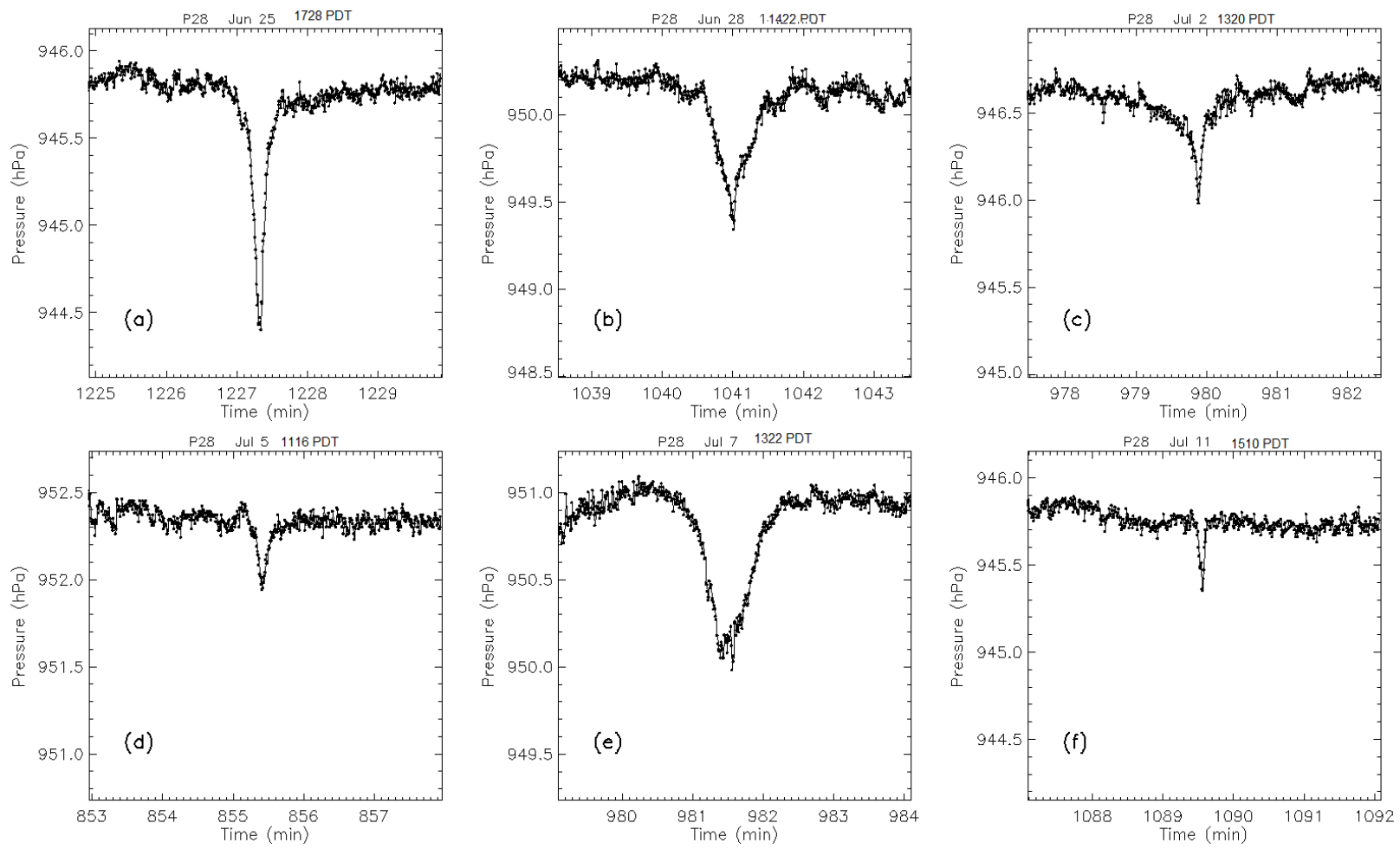

Fig 3. Class A events, considered definite vortex signatures. They are distinct and continuous. Some examples (e.g. (c)) are appreciably asymmetric (see Lorenz 2012b), while others (e.g. (b,e)) have a shape 
that differs from the classical Lorentzian. The time axis is in minutes from the start of the data file; the title of each plot indicates the approximate local time (Pacific Daylight Time) of the centre of the plot.
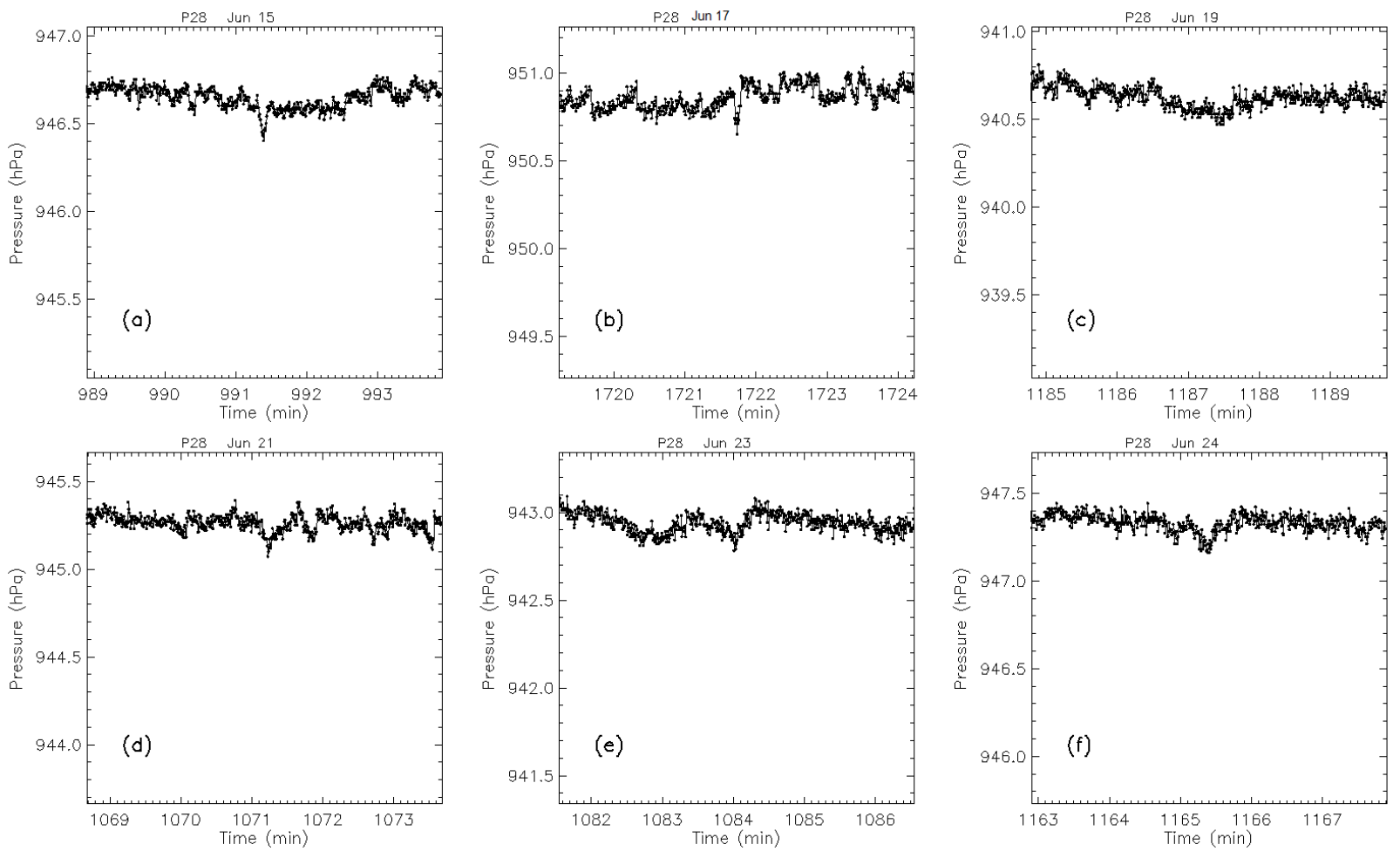

Fig 4. Class B events - these are events considered to be probable vortex passages. The examples (a,b) are borderline class A but relatively small in amplitude and short in duration. Example (c) is rather broad. Examples (d,e) have multiple excursions - which might indeed be other vortices or repeat encounters with the same vortex.

The type A events are often symmetric, but not always (see also Lorenz, 2012b). The pressure trace follows a clear downward trend which then reverses and we note that there are no positive-going counterparts to these steep pressure dips. The largest dip observed in our data is about $1.5 \mathrm{hPa}$, and most have a characteristic width of about 0.5-1 min. Type B events in some cases resemble type A, but are less certain as they are of an amplitude comparable with the noise (in principle some kind of filtering or smoothing of the data might improve discrimination of these cases). In other cases, the trace has a background trend that makes a dip appear unsymmetric: again, on a case-by-case basis, one could fit a curve to the local trend and subtract it to restore the character of the dip. In other cases, the dip resembles an uncorrected (or imperfectly corrected) sawtooth error resulting from improper temperature compensation of the sensor. In yet others, a dip may be steep-sided (i.e. U-shape rather than the V-shape more typical of vortices) and/or accompanied by an adjacent rise, perhaps suggesting a wave or the upwelling/downwelling structures in the convection pattern being advected across the sensor.Type $\mathrm{C}$ events are those designated in the initial pass through the data which closer examination does not strongly 
suggest a devil. Dust devils may well actually be present in some $\mathrm{C}$ events, but these are not included in the population statistics we develop later.

A noise level in the data was estimated from the average standard deviation in pressure readings over 1min intervals centered 2 min before and 2 min after the suspected feature. The magnitude of the pressure drop divided by this deviation gives a signal-to-noise ratio $(S N R)$ of the detection. Histograms of $S N R$ for the three manual classifications are quite distinct (Fig. 9)

\section{Class A : Definite vortices}

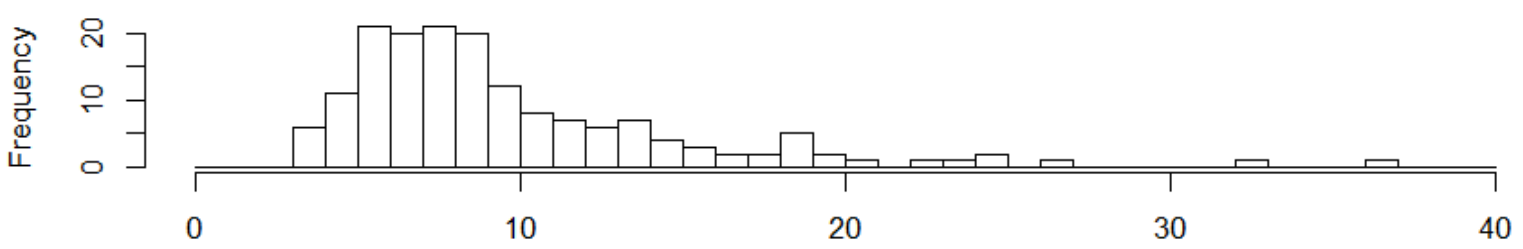

Class B : Probable vortices

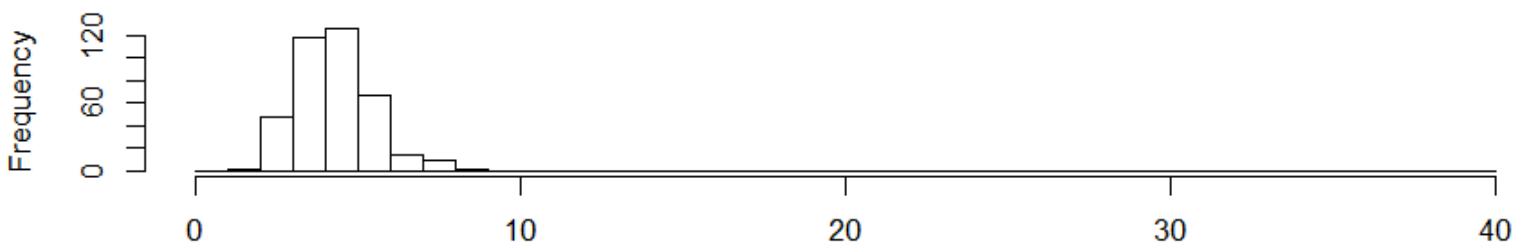

Class C : Possible vortices

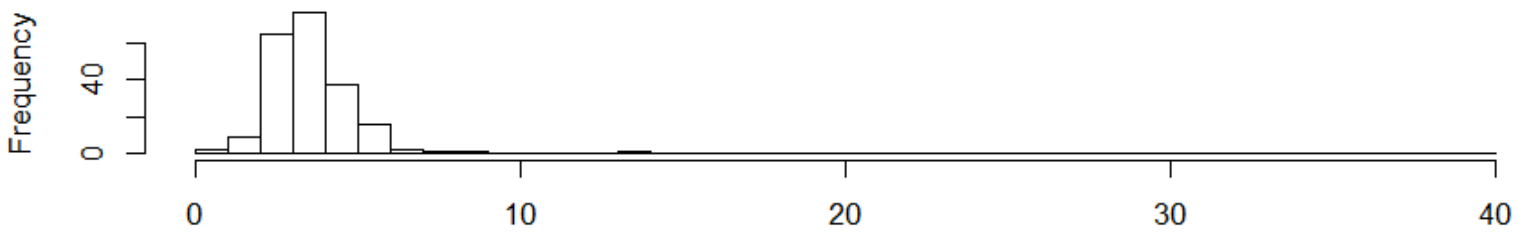

SNR

Fig 5. Histograms of the number of events in the three classes recorded on all three stations as a function of $S N R$ (signal-to-noise ratio - i.e. peak pressure drop divided by the rms scatter in measurements in the minutes adjacent to the event.) There is good discrimination between the class $\mathrm{A}$ and class $\mathrm{C}$ events. A threshold $\mathrm{SNR} \approx 5$ will select almost all Class A events and almost no Class $\mathrm{C}$, with some of the intermediate Class B events. 
Note that the sensor noise is not simple additive white Gaussian noise. A histogram of point-to-point transitions in a long record (not shown) shows a roughly Gaussian shape with a standard deviation of $\approx 3$ $\mathrm{Pa}$. However, the wings are broader than Gaussian, possibly due to digitization noise. It is possible that more sophisticated modeling of these noise contributions might lower the detection threshold for vortices in future work with these same measurement systems. The data presented here, together with the typical event duration of 0.5-1 min, will facilitate the formulation of automatic detection algorithms for processing the large amount of data in future similar surveys.

\section{Dust Devil Events and Conditions}

The number of events identified in the three classes in the three stations are noted in Table 1. It is notable that station P11 nearest the centre of the playa (see Fig. 1) has the largest number of significant events (A,B). The lower count on P10 may reflect that devils have had less time to develop at the south of the playa (usually, the upwind side). Full tabulations of properties of the events, and the raw data files from which readers may reproduce plots such as those in Figs 3 and 4, are given online at (http://www.lpl.arizona.edu/ rlorenz/dustdevils.html).

Table 1. Total number of events June 11- July 11 (31 days)

\begin{tabular}{llllll}
\hline & Class A & Class B & Total $(\mathrm{A}+\mathrm{B})$ & Class C & Total (all) \\
\hline P10B & 33 & 98 & 131 & 61 & 192 \\
P11 & 81 & 162 & 243 & 65 & 308 \\
P28 & 53 & 123 & 186 & 83 & 259 \\
\hline
\end{tabular}

The entire measurement period (Fig. 6), with the possible exceptions of a couple of days, appear to have the classic conditions considered conducive to dust-devil formation, namely strong surface heating and modest windspeeds. Indeed, dust devils are observed more or less throughout. 
Submitted to Boundary Layer Meteorology, 13 January, 2014. Accepted 15 July 2014
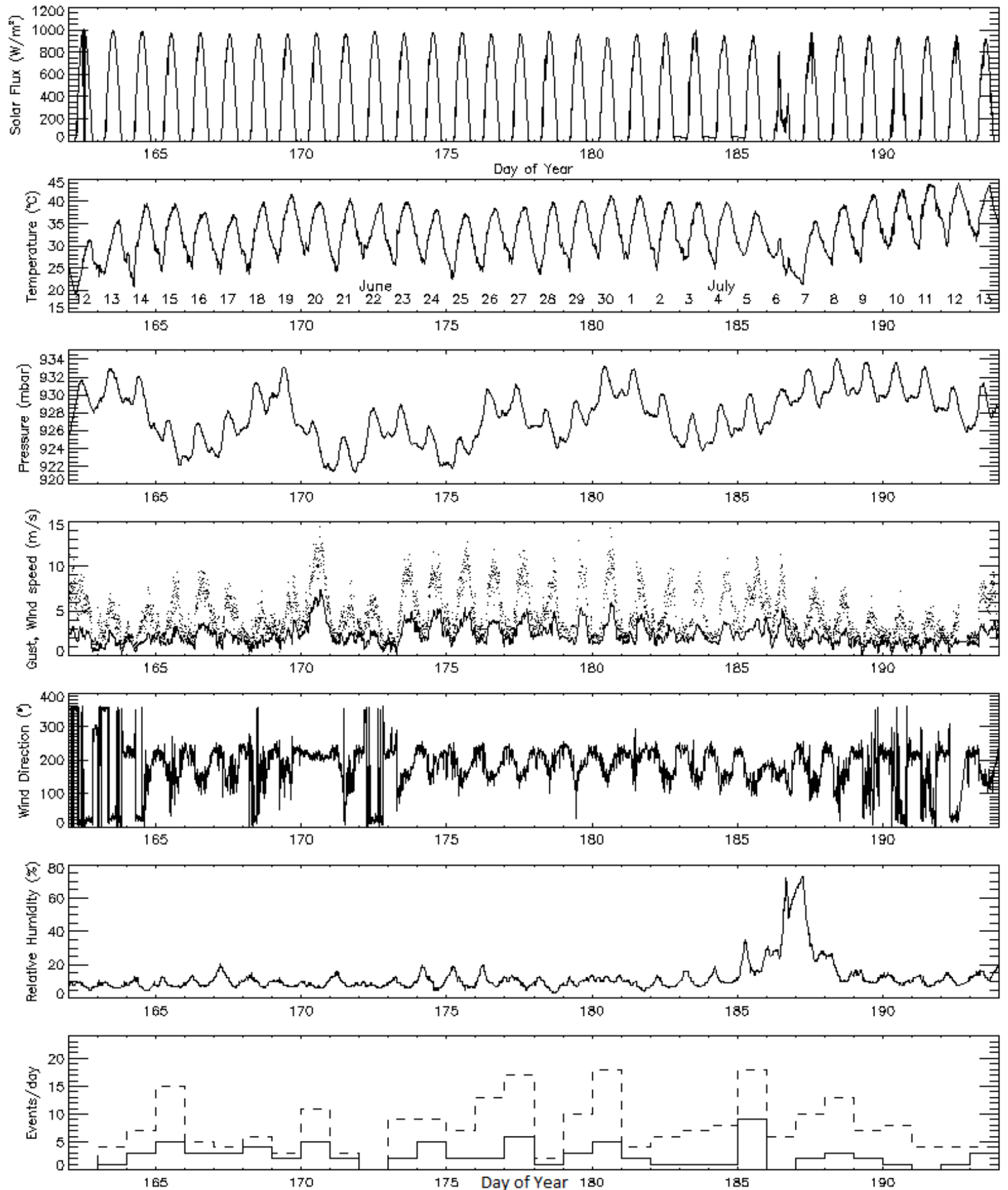
Submitted to Boundary Layer Meteorology, 13 January, 2014. Accepted 15 July 2014

Fig 6. Data from the CMP04 Boulder City meteorological station for the survey period in June/July 2012. The $x$-axis is the day-of-year. The solar flux history shows that skies were almost invariably clear, except on DOY 186 . Wind speed averages $3-5 \mathrm{~m} \mathrm{~s}^{-1}$ during the day, with gusts to typically $\approx 12 \mathrm{~m} \mathrm{~s}^{-1}$. The bottom panel indicates the number of Class $\mathrm{A}+\mathrm{B}$ (dashed line) and Class A only (solid) vortex events recognized in the pressure record (summed for all three loggers). Typically 2-4 Class A events are seen, or roughly one per station per day.

As seen in Fig. 6, events occur throughout the survey period, with no major drop in activity. An inspection of the time of day of occurrence (see Fig. 7) shows the familiar peak of activity towards the early afternoon. The fact that the distribution of events with local time is similar to that documented visually for dust devils (e.g. Balme and Greeley, 2003; Sinclair, 1973) supports the idea that these pressure events are dust-devils or at least dust-devil-like columnar convective vortices. The dip at 1400 hrs PDT may not be significant. It is notable that events occur after 1800hrs PDT, when activity might traditionally thought to cease.

Class A

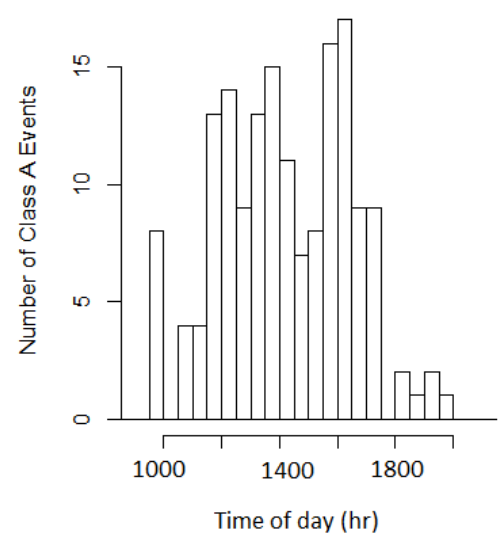

Class B

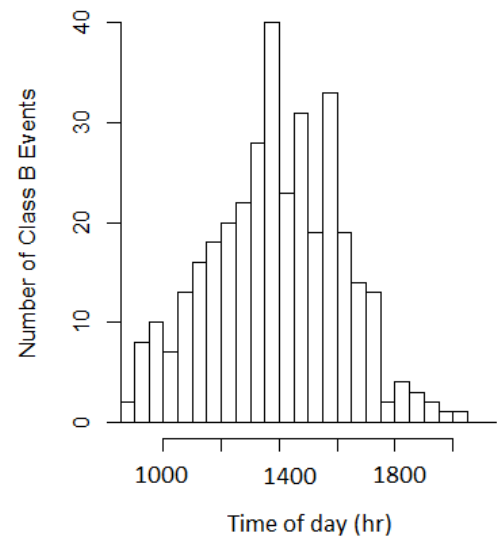

Class $A+B$

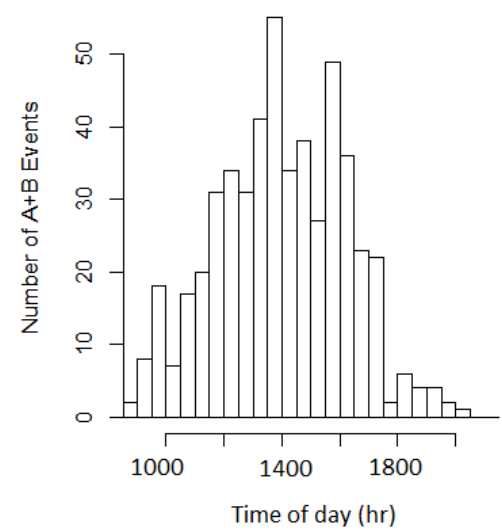

Fig 7. Time of day Statistics from all three loggers combined for class A (A) and class B (B) events. The dip around $1300-1400 \mathrm{hrs}$ PDT is only 1-sigma and thus may not be significant, although Sinclair (1969, his Fig. 6) found a similar double peak. Dust devil chasers need not arrive before about 10:00 ; if they leave at $1600 \mathrm{hrs}$ PDT they may miss about $10 \%$ of events, however.

It has been noted in previous work (e.g. Carroll and Ryan, 1970) that dust-devils may have a certain periodicity in their occurrence (their Fig. 1 shows a record with a pronounced periodicity of about 45 minutes). Since they are sporadic phenomena, any periodicity is only inefficiently detectable. When occurrence times are chosen at random from a Gaussian distribution centered at $1 \mathrm{pm}$ with a deviation of $150 \mathrm{~min}$ (i.e. resembling the observed time distribution in Fig. 7), the interval between events follows a wide distribution, with e.g. 100 min being little less probable than 20-50 min. However, the observed 
distribution of intervals (Fig. 8) is rather broader, and may show some periodicity, with a 'fundamental' of about $20 \mathrm{~min}$ and a notable peak for $40-50 \mathrm{~min}$. Renno et al. (2004) find a $\approx 20$ min periodicity in ground temperature (their Fig. 5) during dust devil conditions although they attribute this to a radiative feedback, whereas a periodicity in surface wind stress and thus convective heat removal seems perhaps more likely. The period probably reflects the advection timescale of the characteristic cell size in the boundary layer convection pattern since devils tend to appear at the upwelling corners of such cells (e.g. Spiga, 2012; Frisch, 1976; Kanak, 2005). Fig. 2 shows several events in close proximity with a spacing of 10-20 min. In future work we will examine this question in more detail using a variety of detection thresholds and statistical approaches : introducing Class B events may detect weak vortices and perhaps convection cell edges without vortices.

P10B, Class A+B

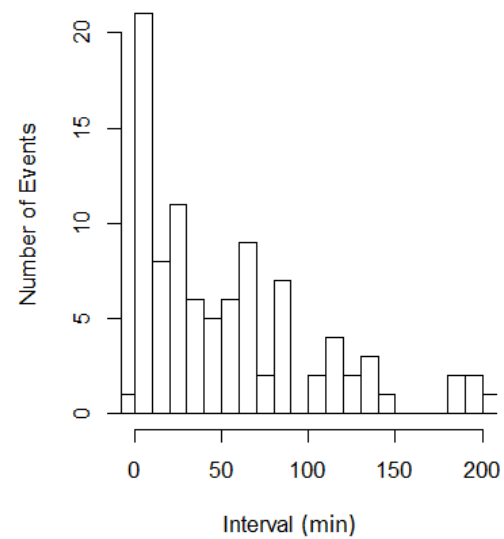

P11, Class A Only

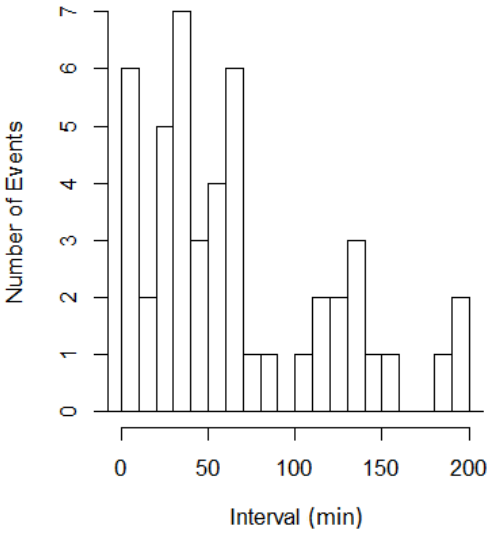

P28, Class A+B

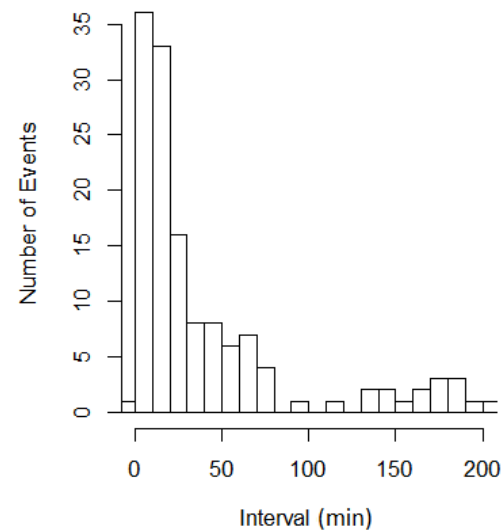

Fig 8. Interval between events. The distribution is more skewed than that for a set of times chosen randomly, suggesting that events are correlated. There are peaks at 20-40 min and 50-70 min. The periodicity may be stronger in a plot of Class A events only (shown for P11) than in $A+B$, but the counting statistics are poorer.

In Fig. 9, following Ellehoj et al. (2010) we plot $\Delta P$ against the event duration. As might be expected, short and shallow events dominate, but the point cloud has two arms from that cluster, a short-but-deep branch driven by close encounters with small devils, and a broad-but-shallow branch due to distant encounters. The exact distribution of points (and in particular how empty the upper-right broad-and-deep corner is) will depend on the relation of pressure drop to dust devil diameter, but this question must be explored numerically with a larger dataset. 


\section{Class A}

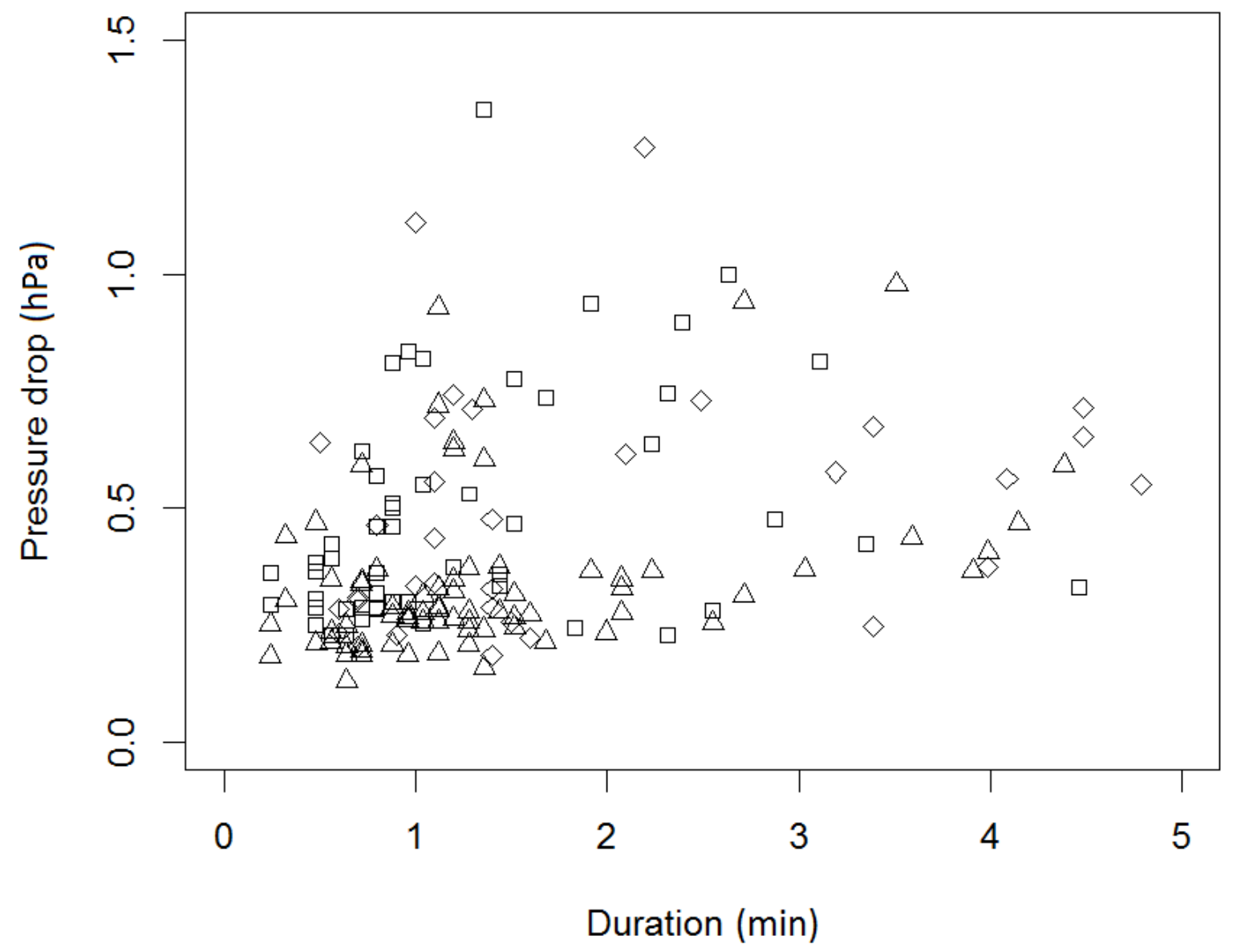

Fig 9. The pressure drop vs duration for the Class A events from all three loggers (P10B diamonds; P11 squares; P28 triangles). As noted in a similar plot for Mars observations (Ellehoj et al., 2010), there are many small, short events (small devils passing close to the logger), and a few large, short events (large devils also making close encounters). A population of modest drops with long durations corresponds to distant encounters with large devils. There are few large, long drops.

The overall size distribution in terms of peak pressure drop is shown in Fig. 10. The cumulative distribution has a slope a little steeper than -1, except at the largest end where the number of events falls off more steeply, perhaps (Lorenz, 2011) because the survey is not long enough to detect the underlying population. It can be seen that the Class $\mathrm{B}$ events follow roughly the same function, but propagate to smaller sizes (down to $\approx 0.15 \mathrm{hPa}$ ). When a threshold of $0.25-0.3 \mathrm{hPa}$ is adopted, almost all events are 
Class A, suggesting this may be a useful threshold for automatic searches in that this will have a low false detection rate. In our survey, $0.3 \mathrm{hPa}$ events occur about once per day on average.

Even considering Class A events only, the number of events detected per day is 5-10 times higher (Fig.10) than the only comparable survey in the literature to date, that of Lambeth (1966). This survey used chart recorders triggered by visual observation of dust devils, and appears to have a much lower detection efficiency (assuming the same underlying frequency of events), either due to a reluctance to trigger consumable chart recording for very small events, and/or an abundance of undetected ('dustless') devils. The population shape function in our survey is similar to that of Lambeth (1966) although those data were for the greatest drop measured in an array of six stations and is thus not strictly comparable with our point measurement.

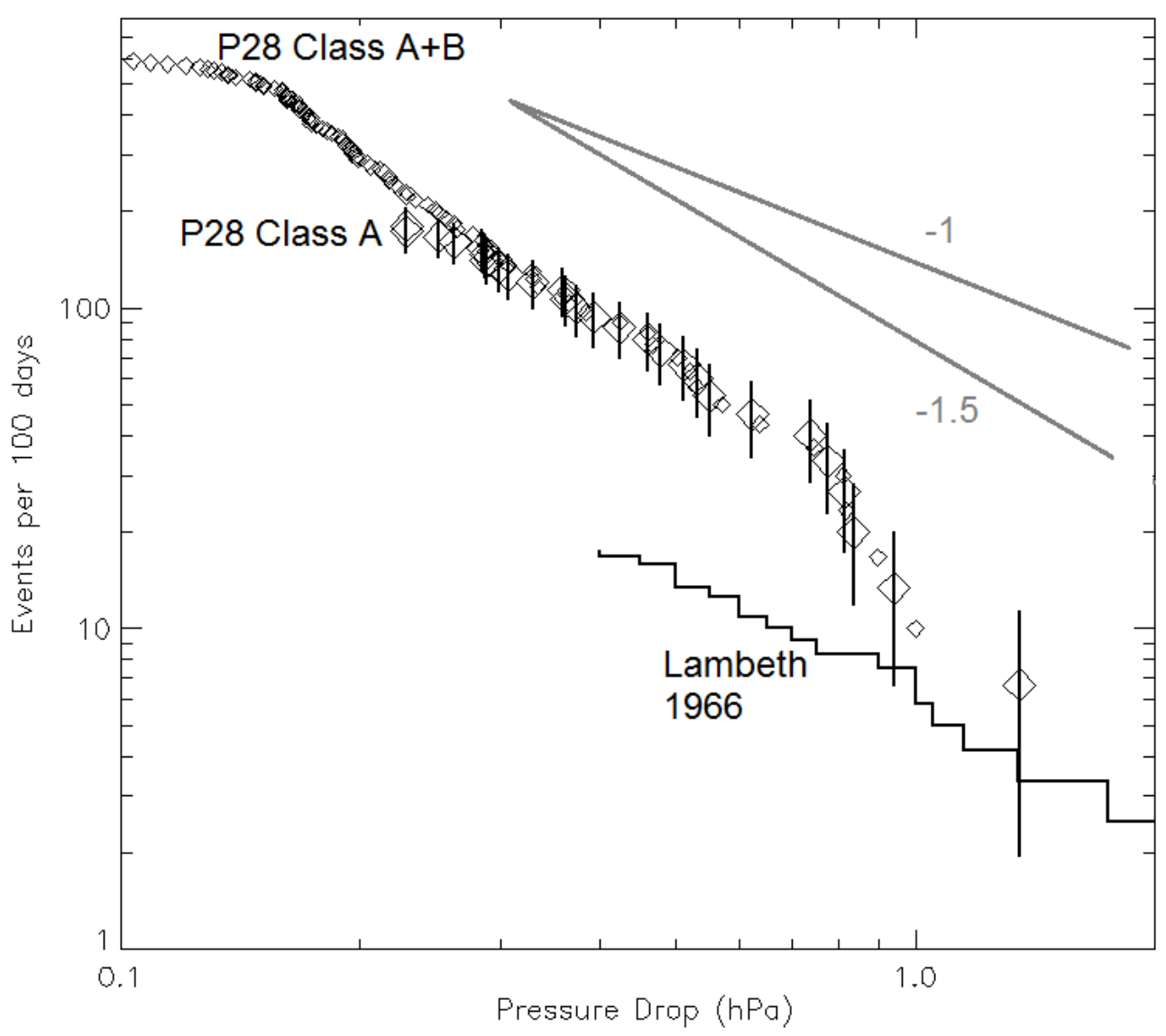

Fig 10. Diamonds are the P10B record at El Dorado, with large symbols corresponding to Class-A events only, and small symbols representing the sum of Classes A+B. It is seen that for events $>0.3 \mathrm{hPa}$, the contribution of class B events is minimal, and we can consider the population assessment robust, i.e. down to that threshold all recorded events are pretty certain and thus the detection efficiency is likely near unity. Between 0.2 and $0.3 \mathrm{hPa}$, Class B events contribute about half the total, so efficiency is likely 
Submitted to Boundary Layer Meteorology, 13 January, 2014. Accepted 15 July 2014

declining to $50 \%$ or less. The $\mathrm{A}+\mathrm{B}$ counts follow the same function slope down to $0.2 \mathrm{hPa}$, which is encouraging. The lines are -1 and -1.5 cumulative power laws - for $0.3-0.8 \mathrm{hPa}$ the data are welldescribed by a -1.5 law. Our survey shows a similar slope to the Lambeth et al. (1966) detections (stepped line) but find a factor of 5-10 more events. Vertical lines are 1- $\sigma$ (i.e. $\sqrt{ } \mathrm{N}$ ) counting errors.

There has been some debate in the literature (e.g. Lorenz, 2011; Pathare et al., 2011; Kurgansky, 2012) whether dust devil diameters follow a power law or an exponential distribution. Similar debates appear regarding rain cell size, convective cloud systems and many other phenomena (see e.g. Newman, 2005 and references in Lorenz, 2012a); the debate is usually clouded by difficulties at the small size end of the population (due to low detection efficiency) and at the large size end (due to poor counting statistics). Several Mars datasets, and one terrestrial one, appear to have fairly robustly straight distributions in a loglog plot (suggesting a power law in diameters) whereas most terrestrial datasets are too poor sparse or too coarsely binned to be conclusive. Plotting data as cumulative distributions, as we have done here, avoids the need for binning. As for pressure drops, the counting statistics in Lambeth et al. (1966) are too poor to be conclusive, as discussed in Lorenz (2012a) but the Mars surveys are well described by -2 differential power laws (i.e. -1 power cumulative).

Our larger dataset now allows us to probe this question more closely for a terrestrial dust devil population. In fact, a -1.5 cumulative slope (i.e. a -2.5 differential power law) describes the P28 population very well from 0.3 to $0.8 \mathrm{hPa}$, where statistics are robust (see counting error bars in Fig. 10) and detection efficiency is high. Above $0.8 \mathrm{hPa}$, there appears an appreciable fall-off $(\mathrm{a}-4$ or -5 power law describes this). Whether a broken power law is an appropriate description (which one might attribute to some kind of threshold phenomenon), or whether a more convex function such as a continuous exponential should be preferred, is in part an aesthetic choice. However, it does seem certain that a simple power law is not a complete description of the terrestrial data.

It should be borne in mind that these are observed pressure drops, not necessarily the peak pressure drop at the core of each devil. A numerical model study is needed to address this relationship. Such a study can be informed by the duration/drop statistics shown in Fig. 9, and will need to use a function of pressure drop vs. distance in a vortex. While an idealized Rankine model (which is conceptually useful, but not accurate) has been invoked in previous work, more realistic models such as the Burgers-Rott or Vastitas (e.g. Vastitas et al., 1991) should be used - these show, for example, that while the pressure drop at the 'wall' of the vortex, where the circumferential speed is a maximum, is about half of the central pressure drop, a drop of about $10 \%$ of that in the core may be seen about three wall diameters away from the center. Thus a pressure field being advected across the sensor may produce an observable dip of $1 \mathrm{~min}$ duration, which corresponds in an ambient wind of $\approx 4 \mathrm{~m} \mathrm{~s}^{-1}$ to a vortex perturbation extending across some $240 \mathrm{~m}$. However, this may correspond to a dust devil of only $40 \mathrm{~m}$ in diameter, which is quite consistent with field observations (e.g. Pathare et al., 2010).

\section{Comparison with Mars}

A direct comparison of absolute pressure drops in dust devils on Mars and Earth is not appropriate, since the background pressures (and therefore the atmospheric density, and thus the dynamic pressure for a given windspeed) differ by almost two orders of magnitude. A proportional pressure drop, however, is a 
useful metric. As well as having an intrinsic appeal, the quantity $\triangle P / P$ can be related approximately to the rotational windspeed, in that the cyclostrophic balance in a vortex structure relates the windspeed and radius to the pressure gradient. As discussed in Lorenz (2012a) to a first order, $\Delta P \sim \rho V_{c}^{2}$, where $\rho$ is the air density and $V_{c}$ the circumferential windspeed. For an ideal gas, $P=\rho R_{o} T / M$, with $T$ the absolute temperature, $M$ the relative molecular mass, and $R_{o}$ the universal gas constant $8314 \mathrm{~kJ} / \mathrm{K} / \mathrm{mol}$ and thus the normalized pressure drop $\triangle P / P \approx\left(R_{o} T / M\right) V_{c}^{2}$. Whereas the density is two orders of magnitude different between earth and Mars, the prefactor in the normalized pressure drop expression differs only by about a factor of two : on Earth, with $T \approx 300 \mathrm{~K}, M \approx 29$, the prefactor is almost double that on Mars, with $T \approx 250 \mathrm{~K}, M \approx 44$, and thus the circumferential windspeed for a given $\Delta P / P$ is only about $41 \%$ larger. A coherent vortex is defined by the circumferential windspeed being appreciable compared with the background wind. Thus the vortex-defining property is, certainly within a factor of two, related to $\triangle P / P$, and thus we plot this quantity in Fig. 11.

The observed Mars events extend to higher normalized pressure drops $(\approx 0.5 \%)$ than those observed in our survey $(0.15 \%)$, suggesting perhaps that Mars dust devils can reach higher intensity than those on Earth (consistent with Martian dust devils ascending to higher altitudes in the thicker Martian boundary layer). The terrestrial counts are generally within a factor of two for the different stations, and suggest an event rate of about five times smaller than for the two Mars populations. 


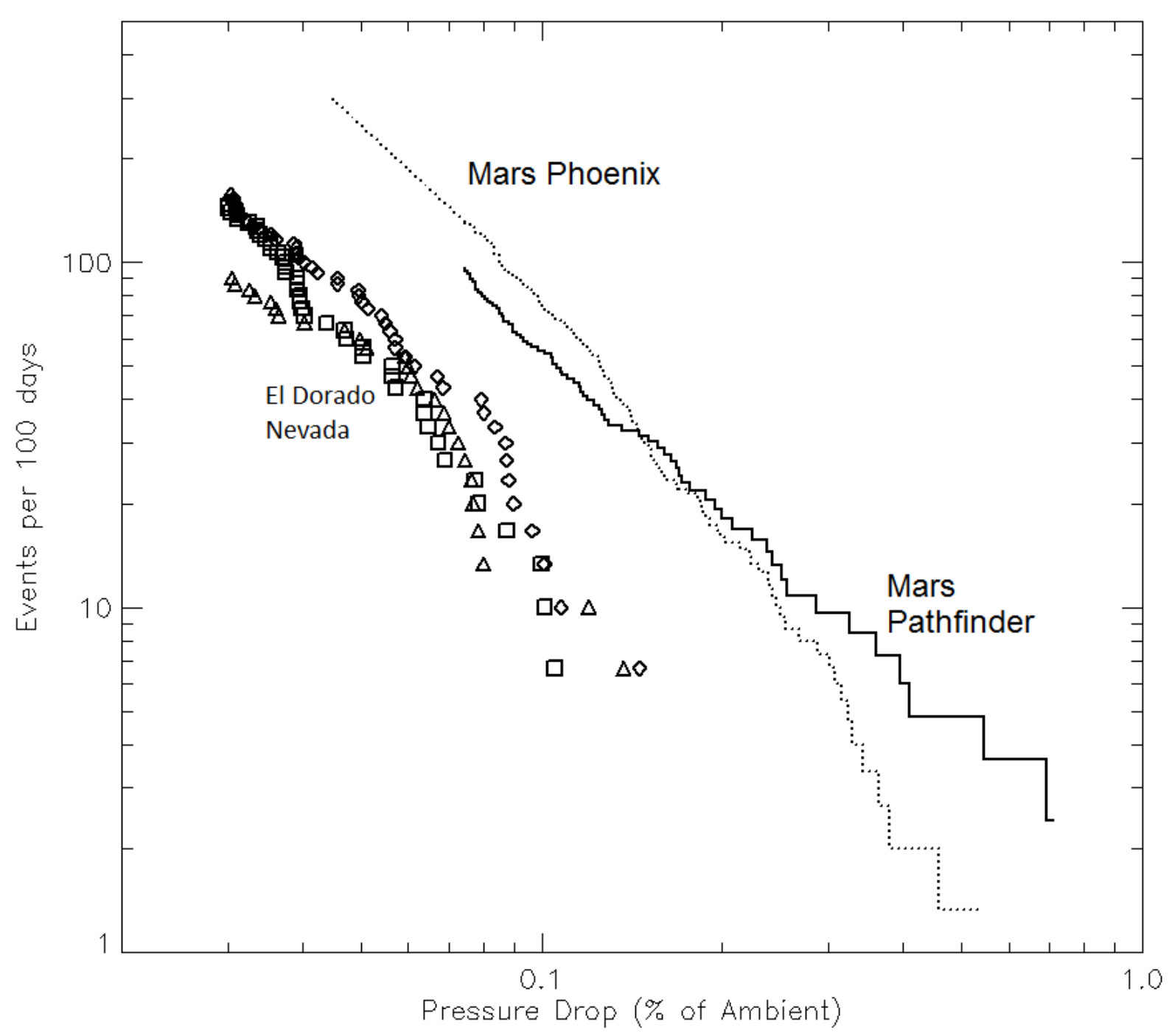

Fig 11. Cumulative pressure drop statistics expressed as a percentage of the ambient pressure. Lines show Mars Pathfinder (79 events $>5 \mathrm{~Pa}$, solid line, Murphy and Nelli, 2002) and Mars Phoenix $(\approx 150$ catalogued events $>5 \mathrm{~Pa} ; 502$ total events $>3 \mathrm{~Pa}$, Ellehoj et al., 2010). Populations recorded by the three terrestrial stations (Class A events only) are nearly coincident, although in the range $0.06 \%<\Delta P / P<$ $0.09 \%$, station P10B (diamonds) appears to see more events by a factor of a few than P11 (squares) or P28 (triangles). Overall the terrestrial counts are about 5 times smaller than the Mars populations. It may not be coincidental that the smallest Martian events identified have a similar threshold, since features on both worlds may be defined by circumferential winds being above ambient.

Pressure thresholds for detection of dust devil vortices have been defined entirely empirically in previous Mars studies, and here (see Fig.5). One useful parameter on which to base a theoretical detection threshold would be the convective velocity scale (Deardorff, 1970) which can be written $w_{*}=$ $\left[(g / T) z\left(F / \rho c_{p}\right)\right]^{0.33}$, where $g$ is acceleration due to gravity, $T$ the absolute temperature, $z$ the height of the 
convecting layer, $F$ is the sensible heat flux and $c_{p}$ the specific heat. The terms in parentheses represent a buoyancy parameter and the kinematic heat flux respectively.

For typical afternoon conditions in terrestrial $\left(g=9.8 \mathrm{~m} \mathrm{~s}^{-2}, \rho \approx 1.1 \mathrm{~kg} \mathrm{~m}^{-3}, c_{p} \approx 1000 \mathrm{~J} \mathrm{~kg}^{-1} \mathrm{~K}^{-1}\right)$ deserts, with $F \approx 400 \mathrm{~W} \mathrm{~m}^{-2}$ and $z \approx 1 \mathrm{~km}$, we find $w^{*} 2 \mathrm{~ms}^{-1}$, and thus the background pressure fluctuation $\rho w_{*}^{2}$ is around $4 \mathrm{~Pa}$, and expressed as a normalized pressure $\left(\rho w_{*}^{2} / P\right)$ is $0.004 \%$. Our putative vortex detections extend down to $\sim 0.02 \%$, or five times the expected background noise level, and indeed Class A detections have a $S N R$ of 5 or more.

On Mars $\left(g=3.7 \mathrm{~m} \mathrm{~s}^{-2}, T=250 \mathrm{~K}, \rho \approx 0.02 \mathrm{~kg} \mathrm{~m}^{-3}, z \approx 5 \mathrm{~km}, c_{p} \approx 840 \mathrm{~J} \mathrm{~kg}^{-1} \mathrm{~K}^{-1}, F \approx 20 \mathrm{~W} \mathrm{~m}^{-2}\right.$, Michaels and Rafkin, 2002) we find $w \approx 0.5 \mathrm{~ms}^{-1}$ and $\rho w_{*}{ }^{2}$ is only $0.005 \mathrm{~Pa}$. Expressed as a normalized pressure drop $(\triangle P / P$, with $P \sim 600 \mathrm{~Pa}$ ) this background level is $\sim 0.001 \%$. A coherent vortex structure must have a pressure perturbation several times this value. Michaels and Rafkin (2002) recognize vortices in their large eddy simulation results with drops up to $\approx 2 \mathrm{~Pa}$, or $\Delta P / P \approx 0.3 \%$, quite comparable with those observed (Fig.11).

The measured pressure drops normalized to a convective pressure perturbation $\Delta P_{c}=\rho w_{*}^{2}$ for Earth and Mars are plotted in Fig. 12. It is seen that the Mars drops are typically an order of magnitude larger than Earth's by this metric, although the interpretation of this is not obvious. 


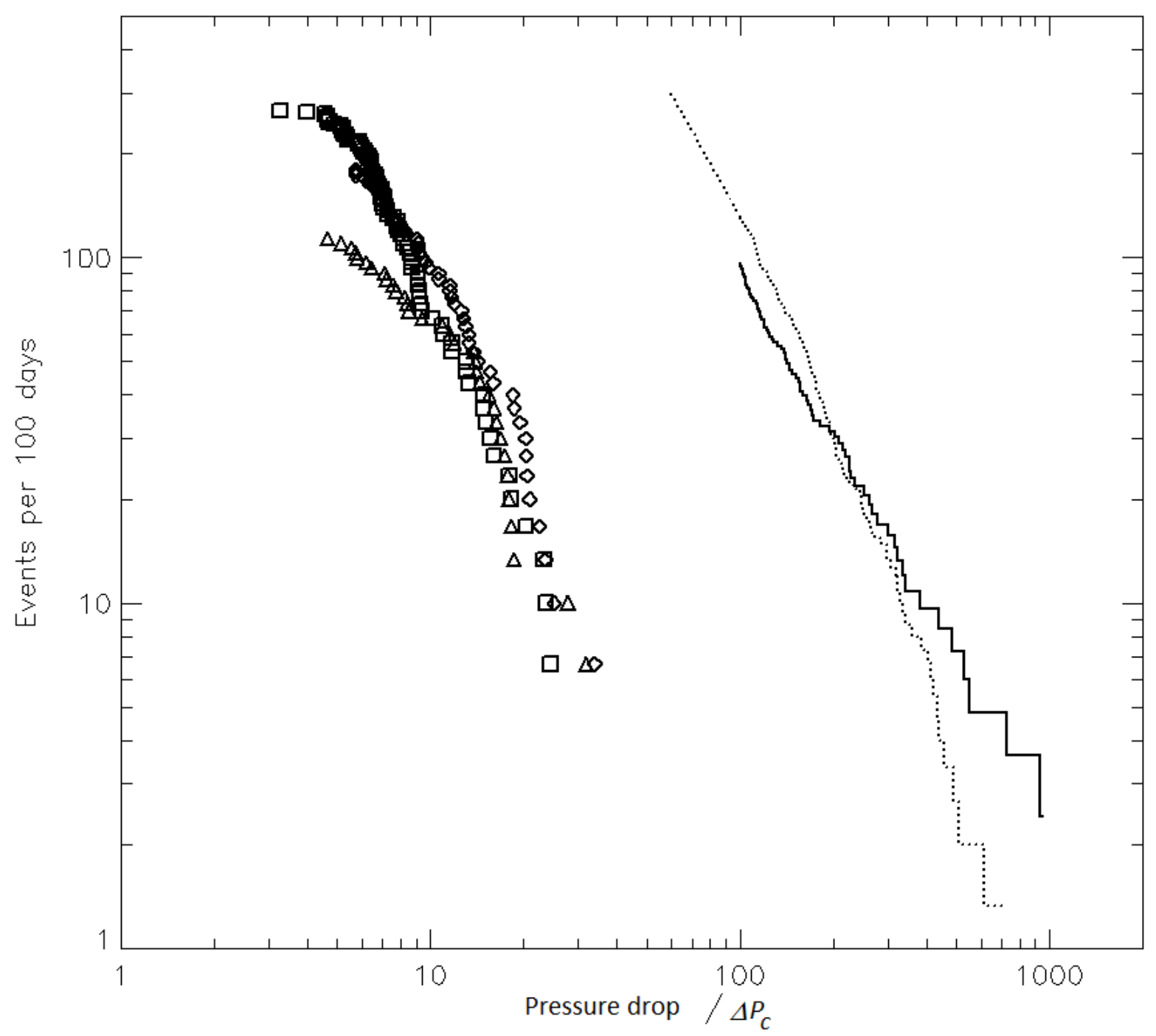

Figure 12. Mars (Phoenix, dotted line and Pathfinder, solid line) and Earth (symbols as in Fig.11) vortex pressure drops normalized to $\Delta P_{c}$, the dynamic pressure associated with the convective velocity scale. Martian devils are an order of magnitude more intense by this metric.

\section{Conclusions}

We have obtained and examined a set of month-long high-resolution pressure records on a desert playa in June-July 2012, under conditions when we would expect dust devils to appear. The records reveal each station sees about one well-defined vortex $(0.3 \mathrm{hPa})$ day, with a couple of less certain detections of smaller events. When expressed as a normalized pressure drop (proportional to circumferential windspeed) the rates for the terrestrial study site are $\approx 40$ per 100 days (i.e. $\approx 3$ per week) for $0.06 \%<$ $\Delta P / P<0.09 \%$, or about a fifth of the rate for Mars. Vortices are recognized robustly with pressure drops 
exceeding about 5 times the observed background noise level, which corresponds reasonably well with the dynamic pressure associated with the convective velocity scale.

The population function appears slightly convex in a log-log plot, and can be fit with a broken power law or an exponential. Inspection of interval histograms suggests devils occur in a correlated manner : in the period 1200 to 1800 hrs PDT where we see most activity, a dust devil is 2-3 times more likely (Fig. 9) to appear in the hour after another dust devil has been observed, than in an hour chosen at random. This correlated appearance is likely associated with a sustained period of favourable boundary layer convection structure, presumably wherein dust devils tend to appear in numerical simulations at the corners of regularly-spaced upwelling sheets (e.g. Hess and Spillane, 1990; Ghenanyi and Taylor, 2011; Kanak, 2005; Toigo et al., 2003; Spiga 2012) that are advected in the ambient wind.

The survey approach described in this paper, of small dataloggers left unattended for long periods, can develop large numbers of candidate dust devil vortex features, which will permit robust statistical analyses. Further surveys would benefit from additional documentation such as from timelapse cameras or co-located wind or dust measurements, and arrays of sensors might explore the horizontal structure of dust devils. Efforts in these directions are underway.

Acknowledgements This work was funded by NASA through the Mars Fundamental Research Program grant number NNX12AI04G. Brian Jackson and Jani Radebaugh are thanked for assistance in the field. The editor and referees made a number of suggestions which improved the manuscript, notably in suggesting the use of the convective velocity scale. 


\section{References}

Balme M, Greeley R (2006) Dust Devils on Earth and Mars. Rev Geophys 44:RG3003

Balme MR, A Pathare A, Metzger SM, Towner MC, Lewis SR, Spiga A, Fenton LK, Rennò NO, Elliott HM, Saca FA, Michaels TI, Russell P, Verdasca J (2012) Field measurements of horizontal forward motion velocities of terrestrial dust devils: Towards a proxy for ambient winds on Mars and Earth. Icarus 221:632-645

Balme MR, Hagermann A (2006) Particle lifting at the soil-air interface by atmospheric pressure excursions in dust devils. Geophys Res Lett 33:L19S01 doi:101029/2006GL026819

Carroll JJ and Ryan JA (1970) Atmospheric Vorticity and Dust Devil Rotation. J Geophys Res 75:5179-5184

Deardorff JW (1970) Convective Velocity and Temperature Scales for the Unstable Planetary Boundary Layer and for Rayleigh Convection, J Atmos Sci 27:1211-1213

Ellehoj MD, Gunnlaugsson HP, Taylor PA, Kahanpää H, Bean KM, Cantor BA, Gheynani BT, Drube L, Fisher D, Harri A-M, Holstein-Rathlou C, Lemmon MT, Madsen MB, Malin MC, Polkko J, Smith PH, Tamppari LK, Weng W, Whiteway J (2010) Convective vortices and dust devils at the Phoenix Mars mission landing site. J Geophys Res 115:E00E16 2010

Frisch AS, Chadwick RB, Moninger WB, Young JM (1976) Observations of Boundary-Layer Convection Cells Measured by Dual-Doppler Radar and Echosonde and by Microbarograph Array. Boundary-Layer Meteorol, 10:55-68

Furstenau S (2006) Solar heating of suspended particles and the dynamics of Martian dust devils. Geophys Res Lett 33:L19S03

Ghenanyi B, Taylor P (2011) Large Eddy Simulation of typical dust devil like vortices in highly convective Martian boundary layers at the Phoenix lander site. Planet Space Sci, 59, 43-50

Hess GD, Spillane KT (1990), Characteristics of dust devils in Australia, J Appl Meteorol, 29: 498-507

Johnson NK (1929) Atmospheric Oscillations shown by the Microbarograph. Q J RMeteorol Soc 55:19-28

Kanak K (2005) Numerical simulation of dust devil-scale vortices. QJ R Meteorol Soc 131:1271-1292

Karstens CD, Samaras TM, Lee BD, Gallus Jr WA, Finley CA (2010) Near-Ground Pressure and Wind Measurements in Tornadoes. Mon Weather Rev 138:2570-2588

Kurgansky M (2012) Statistical Distribution of Dust Devils. Icarus 219:556-560

Lambeth RL (1996) On the Measurement of Dust Devil Parameters. Bull Amer Meteorol Soc 47:522-526

Lorenz RD (2011) On the Statistical Distribution of Dust Devil Diameters. Icarus 215:381-390

Lorenz RD (2012a) Pressure Drops in Dust Devils: Earth and Mars. Planet Space Sci 60:370 375 
Lorenz RD (2012b) Observing Desert Dust Devils with a Pressure Logger. Geosci Instrum Meth Data Systems 1:209-220

Lorenz RD (2013) Irregular Dust Devil Pressure Drops on Earth and Mars: Effect of Cycloidal Tracks. Planet Space Sci 76:96-103

Metzger S, Balme MR, Towner MC, Bos BJ, Ringrose TJ, Patel MR (2011) In situ measurements of particle load and transport in dust devils. Icarus 214:766-772

Michaels T and Rafkin SCR (2002) Large eddy simulation of atmospheric convection on Mars. QJR Meteorol Soc, 128:1-25

Murphy J, Nelli S (2002) Mars Pathfinder convective vortices: Frequency of occurrence. Geophys Res Lett 29:2103

Newman MEJ (2005) Power Laws, Pareto distributions and Zipf's law. Contemporary Phys. 46: $323-376$

Pathare AV, Balme MR, Metzger SM, Spiga A, Towner MC, Renno NO, Saca F (2010) Assessing the power law hypothesis for the size-frequency distribution of terrestrial and Martian dust devils. Icarus 209:851-852

Posey J, Pierce AD (1971) Estimation of Nuclear Explosion Energies from Microbarograph Records. Nature 232:253

Ringrose TJ, Patel MR, Towner MC, Balme MR, Metzger SM, Zarnecki JC (2007) The Meteorological Signatures of Dust Devils on Mars. Planet Space Sci 55:2151-2163

Ryan JA, Lucich RD (1983) Possible dust devils vortices on Mars. J Geophys Res 88:1100511011

Sinclair PC (1973) The Lower Structure of Dust Devils. J Atmos Sci 30:1599-1619

Snow JT McClelland T (1990) Dust devils at White Sands Missile Range New Mexico 1: Temporal and Spatial Distributions. J Geophys Res 95:13707-13721

Spiga A (2012) Comment on "Observing desert dust devils with a pressure logger" by Lorenz (2012) - insights on measured pressure fluctuations from large-eddy simulations. Geosci Instrum Method Data Syst 1:151-154

Toigo AD, Richardson MI, Ewald SP and Gierasch PG (2003) Numerical simulation of Martian dust devils. J Geophys Res (Planets) 108: 5047, doi:10.1029/2002JE002002

Tratt DM, Hecht MH, Catling DC, Samulon EC and Smith PH (2003) In situ measurement of dust devil dynamics: Toward a strategy for Mars. J Geophys Res (Planets) 108:5116,1-7 doi:101029/2003JE002161

Wyett RE (1954) Pressure Drop in a Dust Devil, Mon Weather Rev, 82:7-8 